\title{
Updates in Postoperative Pain Management
}

E.F.Gadalla, E.S.Abdelazim and A.M.Abdrabo

Anesthesia and Intensive care, Dept., Faculty of Medicine, Benha Univ., Benha, Egypt

Email:ahmed.andeel50@gmail.com

\begin{abstract}
In the postoperative period, about 20-80 percent of patients report moderate-to-severe pain. Thermal, mechanical, or chemically caused tissue damage are among the most common causes of postoperative pain. It is mainly nociceptive in nature. The goal of this study is to examine all techniques and medicines used for postoperative pain management, with a focus on all new developments in the treatment of postoperative pain. Methods: As you can see, it's a review of There was a search done using the following media keywords in MEDLINE, Embase, Pubmed, and CINAHL Plus in the same time period from 2000 to 2021: Articles written in languages other than English are not eligible for evaluation. Finally, the outcomes are as follows: When it comes to postoperative pain treatment, opioids are still the go-to option, but the current opioid crisis is driving doctors to explore alternative options that utilise a variety of pain management modalities. Other pain-relieving receptors in the spinal cord are targeted by these pathways instead of opioid receptors in the brain and body. When administered as a single dose, local anaesthetics, whether infiltrated locally or utilised in regional anaesthesia methods, have a short half-life. Extended postsurgical pain treatment using local anaesthetics may be achieved by utilising patient-controlled analgesia pumps or by combining them with other analgesics such as epinephrine, dexamethasone, or clonidine.
\end{abstract}

Keywords: Postoperative, Pain, analgesics

\section{Introduction}

In the case of surgery, trauma or acute sickness, acute pain is the expected physiologic reaction to an unpleasant chemical, thermal or mechanical stimulation. When tissue is damaged, the pain receptors (nociceptors) become activated, resulting in acute pain. Patients often seek medical attention and are hospitalised to hospitals because of pain. In the United States, 46 million people have surgery every year, and as a result, they experience acute surgical pain. [1]

To put it another way, acute pain is described by the American Society of Anesthesiologists as pain that a surgical patient feels following the operation. Relief from pain is now considered a human right by organisations such as the World Health Organization and the International Association for the Study of Pain. Complications and a longer recovery time are possible when postoperative pain is not properly controlled. Acute pain that is not managed may lead to chronic pain and a deterioration in quality of life. Short hospital stays, lower hospital expenses, and higher patient satisfaction are all benefits of effective pain management. Thus, postoperative pain treatment has become an increasingly scrutinised quality metric. Patient satisfaction with in-hospital pain treatment is measured by the Hospital Consumer Assessment of Health Providers and Systems (HCAHPS) ratings, which may have an impact on payments. [2].

When dealing with extreme pain, opioids given intramuscularly as epidurals or IV are helpful. Preventive analgesia, such as nerve blocks, and adjunctive treatment, such as infusion pumps, as well as delivery modalities. Oral opioids are helpful for moderate to severe pain, and tramadol is used for moderate to moderately severe pain because of its effectiveness that is similar to morphine, but it has less severe side effects. NSAIDs that reduce the need for opioid painkillers, such as ketorolac, and NSAIDS that target COX-2 have both been studied for use in the treatment of chronic pain. Nonpharmacologic therapies like physical therapy or cryotherapy may be used in conjunction with a customised analgesic regimen.

Clonidine and dexmedetomidine, which are alpha2 adrenergic agonists, have potent anaesthetic and analgesic-sparing properties. After surgery, premedication with oral and transdermal clonidine reduced the need for PCA-morphine by half. Postoperative pain and opioid analgesic use are both reduced with dexmedetomidine, a pure alpha- 2 agonist [4].

Analgesic sparing characteristics have been claimed for a wide range of nonopioid pharmacologic agents utilised during the perioperative period (e.g., adenosine, droperidol, magnesium, gabapentin). Even though these chemicals' analgesic-sparing properties haven't been well studied, their application for treating acute postoperative pain is still considered experimental [5].

Using multimodal anaesthesia to target different mechanisms of action in the peripheral and central nervous systems, the guidelines aim to provide evidence-based recommendations for better postoperative pain management by combining multiple medications that act on different pain receptors or are administered in various ways [3].

The goal of this study is to examine all postoperative pain management techniques and medicines, with a focus on the most recent changes in how we treat postoperative pain.

\section{Methods}

This is a review paper, and the following media keywords were used in the search in MEDLINE, Embase, Pubmed, and CINAHL Plus within the same time period: From 2000 to 2021, there have been 
articles on (postoperative; pain; analgesics) Articles written in languages other than English are not eligible for evaluation. Postoperative pain; analgesics are important search terms.

\section{Results}

Approximately $\quad 20-80 \%$ patients experience moderate-to-severe pain during their postoperative course. Postoperative pain is mostly nociceptive in nature, with underlying etiologies including thermal-, mechanical-, or chemical-induced tissue damage [6].

Sociologic factors such as poor initial pain assessment, inadequate clinical knowledge by healthcare provider, or the patient's misconception or poorly conveyed expectations can contribute to it. Timely management of acute postoperative pain not only is vital to patient's surgical experience but also saves valuable healthcare resources by decreasing the length of hospital stay, time to discharge, readmission rates, and time before ambulation [7].

Mismanagement of acute postoperative pain can lead to chronic pain in up to $50 \%$ of patients, which has significant personal, social, and economic implications. Pain has many components including physical, psychiatric, and emotional challenges. Successful postoperative pain management warrants a multidisciplinary approach involving both anesthesiologists and surgeons with a balanced use of pharmacologic and regional anesthetic techniques that target several nociceptive receptors and pathways [8].

Though opioids remain the leading modality to manage postoperative pain, the recent opioid epidemic has motivated clinicians to pursue other multimodal pathways for pain management to mitigate the use of large doses of opioids. These pathways target other receptors than the opioid receptors in the spinal cord to mitigate pain [8].

These include local tissue action by inhibiting prostaglandin, bradykinin, and substance $\mathrm{P}$ via $\mathrm{COX}-2$ inhibitors, blocking nerve synapses at peripheral nerve sites with local anesthetics, and targeting other receptors than the opioid in the central nervous system including gabapentinoids, alpha agonists, NMDA antagonists, NSAIDS, and acetaminophen [9].

Drugs such as cyclooxygenase (COX) inhibitors (acetaminophen, non-steroidal anti-inflammatory drugs (NSAIDs), GABA analogs (gabapentin, pregabalin), $\alpha 2$ agonists (clonidine, dexmedetomidine), N-methylD-aspartate (NMDA) antagonists (ketamine), local anesthetics (lidocaine, bupivacaine, ropivacaine)) are part of most ERAS (enhanced recovery after surgery) protocols devised by various healthcare institutions to improve clinical outcomes after surgery [3].

Local anesthetics whether locally infiltrated or used in regional anesthesia techniques have a short half-life when used alone as a single shot. Local anesthetics can be used in continuous nerve catheters using patient-controlled analgesia pumps or combined with additives such as epinephrine, dexamethasone, or clonidine for extended postsurgical pain management, but these are not without limitations. Since repeated administration or using a higher dose of local anesthetics like bupivacaine places patients at increased risk of systemic complications, the search for extended release counterparts continues. Neosaxitoxin, HTX-011, POSIMIR $®$ (sucrose acetate isobutyrate extended-release bupivacaine), and Exparel ${ }^{\circledR}$ (bupivacaine liposome injectable suspension) are some examples of new local anesthetic formulations designed to provide sustained pain relief after surgery [10].

\section{Discussion}

There isn't a perfect analgesic medication out there yet. In the first several hours after surgery, data from single-dose studies on particular patient groups restrict the evidence for employing medicines with a low number required to treat (NNT).

Drug treatments alone should not be sufficient for postoperative pain control. Traditional approaches to major abdominal surgery, such as epidural analgesia or opioid-based intravenous patient controlled analgesia (IVPCA), are associated with superior pain control, but do not translate into better recovery or reduced morbidity when compared with pain management strategies used in an enhanced recovery after surgery (ERAS) pathway [11].

When used correctly, postoperative analgesia is a critical part of most ERAS routes and helps patients heal faster. When feasible, they promote the use of opiate-sparing methods such as regional analgesia and support the use of multimodal analgesia.

When using multimodal analgesia, various medicines are used to target different sections of the body's anatomical pain pathways in order to reduce pain. Drugs that block ascending impulses of pain in the peripheral or central nervous system, while also enabling inhibitory spinal pathways, are often referred to as analgesics. This reduces the transmission of nociceptive impulses and the brain's perception of them as pain. Synergistic effects are produced when various drugs with distinct mechanisms of action are combined. This reduces the burden of side effects associated with single-drug regimens and allows for lower dosages to be used.

According on the kind of surgery, some combinations have more or less of the same elements in them. A cooperation between surgeons and anaesthetists called PROSPECT (PROcedure-SPECific Pain ManagemenT) has produced publications that offer practical evidence-based summaries on procedure-specific pain control. However, they must be customised for each patient, taking into consideration variables like as prior analgesic usage, co-morbidities, pharmacogenomics, epigenetics, medication interactions, and tolerance. [3].

The most current Cochrane review of RCTs on single-dose oral analgesics for acute postoperative pain in adults found an NNT of $3 \cdot 6$ (or $95 \%$ effectiveness). To enhance the effectiveness of paracetamol, it was 
combined with other analgesics, such as ibuprofen or codeine (NNT $1 \cdot 5,2 \cdot 2$ ) or oxycodone (NNT $1 \bullet 8$, respectively).

Prophylactic intravenous paracetamol administration is linked to less postoperative nausea and vomiting, which may be related to better pain management. Concerns about paracetamol's potential hepatotoxicity have been raised, however current research indicates that at therapeutic dosages, this is not likely to occur. In other words, using paracetamol in a multimodal approach may help with pain management and reduce the need for additional analgesics [14].

A multimodal analgesic approach is recommended by the ERAS Society while using non-steroidal antiinflammatory medications (NSAIDs). The use of NSAIDs following major surgery has been shown to decrease IVPCA morphine use. Acute renal damage and anastomotic leak are still potential consequences. There were two studies that looked at the postoperative side effects of NSAIDs. Recent Cochrane reviews of postoperative nonsteroidal anti-inflammatory drugs (NSAIDs) found that the effects of these drugs on renal function remain unclear, in part because of a scarcity of data on how safe these drugs are during the perioperative period.

Anastomotic leak remains a mystery, although more recent research indicates that if there is a link, it is with non-selective NSAID usage. There is still guidance for considering each patient individually due to additional adverse effects such as increased risk of thromboembolic events, gastrointestinal bleeding and worsening of cardiac failure [15] that need to be considered.

Meloxicam, an NSAID long used to relieve pain by reducing inflammation, is one medicine for mild to moderate pain. For this reason, it protects the stomach mucosa while also protecting the renal system: It is more effective against COX-2 than COX-1. It has been proven time and time again to be helpful in the treatment of both acute and chronic pain

\section{Dexamethasone}

One perioperative dosage of dexamethasone has an unknown analgesic effect and side effects. An i.v. dexamethasone dosage was studied to see whether it had any effect on postoperative pain and if there were any side effects. There were 45 trials that comprised 5796 individuals on dexamethasone $1.25-20 \mathrm{mg}$. Two hours after surgery, those getting dexamethasone reported lower pain ratings than those who did not $(\mathrm{MD}=$ mean difference; 95 percent confidence interval: $-0.83,0.15$ ); and 24 hours following surgery, those who did not received dexamethasone reported lower pain scores than those who did;

There was a significant reduction in the use of opioids in patients treated with dexamethasone at 2 hours [MD $0.87 \mathrm{mg}$ morphine equivalents (95 percent confidence interval: 1.40 to 0.33 )] and at 24 hours [MD $2.33 \mathrm{mg}$ morphine equivalents (95 percent confidence interval: 4.39, 0.26)], as well as fewer rescue analgesics for intolerable pain [relative risk 0.80 (95 percent confidence interval: 0.69 , When it came to the opioid-saving impact, there was no dosage response. Dexamethasone did not promote infection or delay wound healing, although blood glucose levels were greater after 24 hours [MD $0.39 \mathrm{mmol}$ litre1 (95 percent CI: 0.04, 0.74)] [16].

\section{Opioids}

In the treatment of moderate to severe acute pain, opioids have long been the standard of care. As soon as three weeks after surgery, $10 \%$ of 379 patients had opioid-related adverse events (ORADE). These events were more frequent in older males with higher ASA fitness grades, those who had numerous comorbid conditions, and those who had a history of alcohol or drug addiction. ORADEs were linked to a $1 \bullet 6$-day increase in hospital stay [17].

Administering painkillers for longer than the recommended time in the hospital poses a danger to patients. An American study of patients receiving chronic opioid therapy found that $27 \%$ began taking opioids after surgery, and a recent systematic review found that less than half of opioid prescriptions issued after surgery are used by the patient after discharge, highlighting a potential source of opioid supply diversion and abuse [17].

For patients who are currently using opioids or benzodiazepines, or who have been diagnosed with substance use disorder or another mental health problem, extended postoperative usage is a significant risk factor. As well as an increased risk of uncontrolled pain, patients who take opiates before surgery have a greater burden of ORADE, particularly respiratory depression and drowsiness. To assist efficient analgesia, limit increasing opioid usage, and lessen opioid-induced hyperalgesia, multimodal analgesic methods are critical in this population.

It is important to keep track of these patients' preadmission dosage of opioids because otherwise, they run the danger of going into withdrawal. Because of opioid tolerance, IVPCA opioids may be beneficial in certain situations, but patients may likely need continuous infusions and larger bolus dosages than opioid-naive patients.

One other way to reduce risks is to rotate or switch opioids, utilise other methods, such as local or regional anaesthesia, and make sure that the patient is discharged with a "reverse analgesic ladder," which gradually returns them to their predmission opioid prescription. There are many advantages to using a continuous central neuraxial block or an EA throughout a variety of surgical procedures. As of now, there's level 1 evidence that analgesia at rest is improved, as well as lower incidences of ileus, pulmonary problems, surgical stress response, and negative nitrogen balance.

A local anaesthetic infusion is often a part of an epidural formulation. Oxycodone is often used in conjunction with opiates such as morphine and buprenorphine. Other common opiates include 
diamorphine, hydromorphone, and tramadol. Clonidine, a less frequent adjunct, prevents sympathetic outflow when administered in neuraxial blocks. After intraabdominal surgery, EA provides moderate pain relief compared to IVPCA opioids, with statistically significant but non-clinically meaningful reductions in pain ratings at rest. Using $\mathrm{EA}$, on the other hand, may improve your chances of making it to DrEaMing faster. By improving food tolerance while decreasing nausea, vomiting, and discomfort, EA decreases paralytic ileus while boosting gastrointestinal transit recovery [20].

Compared to IVPCA opioids, the analgesic failure rate for EA is greater (120 vs 34 in 1000 respectively), and the use of EA is more likely to result in hypotension treatments (120 versus 17 per 1000). Complications are a possibility with this method.. Permanent damage from all EA is estimated at $17 \cdot 4$ (95 percent). Paraplegia or death affects $6 \cdot 1(2 \cdot 2$ to $13 \cdot 3$ ) people per 100,000 , whereas the death rate is $7 \cdot 2$ $(2 \cdot 2$ to $27 \cdot 8)$. Additionally, even though the chance of developing an epidural haematoma is very low, patients on antiplatelet and/or anticoagulant medication should exercise care. In open surgery, current ERAS recommendations suggest thoracic EA; however, for laparoscopic operations, they do not recommend it. Patients having oesophagectomy are advised to use thoracic EA as a first line treatment in ERAS pathways, which seems to be surgery-specific. However, higher-level evidence has been provided for gynaecological ERAS routes for local anaesthetic infiltration of wounds [21].

\section{Analgesia administered via the vein}

This method utilises a local anaesthetic to reduce surgical stress while also administering a long-acting opioid such as morphine or diamorphine, which has analgesic effects that last up to 24 hours after the operation is completed. An optimistic estimate of $2 \cdot 2$ (95 percent) indicates that spinal anaesthesia/analgesia is very effective with a low complication rate. 100000 for long-term damage, and 1 to 3 per 100000 for death or paraplegia [13].

After laparoscopic colonic resection and abdominal surgery for gynaecological cancer, intrathecal analgesia reduces opiate intake and pain ratings when administered in an ERAS programme. However, there is no convincing evidence that it has an effect on hospital stay length. The major worry is the possibility of post-operative delayed respiratory depression. For laparoscopic colorectal surgery, this method is approved by the most current ERAS guidelines (version 22).

\section{Blocks for the abdominal wall}

More and more people are choosing novel abdominal truncal blocks in the last decade, such as transversus abdominis plane (TAP) and rectus sheath blocks As a result of blocking T7-T11, T12, the subcostal nerve, and ilioinguinal and iliohypogastric (L1-L2) nerves, TAP block produces analgesia. An infusion or a single dosage of these blocks may be used for longer-term benefit. Both laparoscopically and with ultrasound imaging, they may be inserted subcutaneously or directly beneath the skin. Truncal blocks often include a local anaesthetic and an adjuvant, although this isn't always the case. The bulk of adjunct research comes from limb blocks, and this essay will not go into depth about it. There are several exceptions to this rule, however, such as morphine, which does not enhance analgesia quality but instead causes more adverse effects, while clonidine, dexamethasone, and ketamine all have undesirable side effects due to systemic absorption [23].

Analgesic equivalence or superiority has been shown, however the diversity in methods restricts the capacity to integrate research data in a meta-analysis. For the first 24 hours after surgery, pain ratings at rest were identical to those for EA (mean difference $0 \bullet 5,95$ percent confidence interval $0 \bullet 1$ to $1^{\circ} ; \mathrm{P}=0 \bullet 10$ ), with less hypotension and a shorter stay in the hospital. For example, incisional local anaesthetic injection is suggested above TAP blocks or thoracic EA ERAS guidelines for perioperative treatment in gynaecological/oncological surgery, whereas TAP blocks are highly recommended as part of multimodal analgesia during minimally invasive colorectal surgery [24].

\section{The use of methods other than medication}

Postoperative pain management may be assisted by non-pharmacological treatments during the perioperative period. These are often low-cost and simple to put into practise. Cognitive behavioural therapy is one kind of preoperative strategy that involves educating the patient and addressing their mental health issues. While undergoing surgery, individuals with anxiety benefit most from the use of distraction methods such as music, aromatherapy, canine therapy, and virtual reality. When utilised in the postoperative context as part of a self-management programme, they may help patients become more selfreliant. Concerns about traditional pharmacotherapies' side-effect load have prompted researchers to examine them in a wider range of situations, including acute postoperative pain [25].

\section{Conclusion}

When it comes to postoperative pain treatment, opioids are still the go-to option, but the current opioid crisis is driving doctors to explore alternative options that utilise a variety of pain management modalities. Other pain-relieving receptors in the spinal cord are targeted by these pathways instead of opioid receptors in the brain and body. When administered as a single dose, local anaesthetics, whether infiltrated locally or utilised in regional anaesthesia methods, have a short half-life. Extended postsurgical pain treatment using local anaesthetics may be achieved by utilising patientcontrolled analgesia pumps or by combining them with other analgesics such as epinephrine, dexamethasone, or clonidine. 


\section{References}

[1] M. F. Cooney . "American Society for Pain Management Nursing position statement with clinical practice guidelines: authorized agent controlled analgesia," Pain Manag. Nurs., vol. 14, pp. 176-181, 2013.

[2] J. R. Hsu, H. Mir, M. K. Wally, and R. B. Seymour, "Clinical practice guidelines for pain management in acute musculoskeletal injury," J. Orthop. Trauma, vol. 33, pp. e158, 2019.

[3] R. Chou ."Management of Postoperative Pain: a clinical practice guideline from the American pain society, the American Society of Regional Anesthesia and Pain Medicine, and the American Society of Anesthesiologists' committee on regional anesthesia, executive committee, and administrative council," J. pain, vol. 17, pp. 131-157, 2016.

[4] B. Tufanogullari. "Dexmedetomidine infusion during laparoscopic bariatric surgery: the effect on recovery outcome variables," Anesth. Analg., vol. 106, pp. 1741-1748, 2008.

[5] A. Fassoulaki, K. Patris, C. Sarantopoulos, and Q. Hogan, "The analgesic effect of gabapentin and mexiletine after breast surgery for cancer," Anesth. Analg., vol. 95, pp. 985-991, 2002.

[6] T. J. Gan, A. S. Habib, T. E. Miller, W. White, and J. L. Apfelbaum, "Incidence, patient satisfaction, and perceptions of post-surgical pain: results from a US national survey," Curr. Med. Res. Opin., vol. 30, pp. 149-160, 2014.

[7] H. Kehlet, T. S. Jensen, and C. J. Woolf, "Persistent postsurgical pain: risk factors and prevention," Lancet, vol. 367, pp. 1618-1625, 2006.

[8] J. Smith. "Enhanced recovery after surgery (ERAS) program for lumbar spine fusion," Perioper. Med., vol. 8, pp. 1-9, 2019.

[9] W. R. Spanjersberg, J. D. P. Van Sambeeck, A. Bremers, C. Rosman, and C. Van Laarhoven, "Systematic review and meta-analysis for laparoscopic versus open colon surgery with or without an ERAS programme," Surg. Endosc., vol. 29, pp. 3443-3453, 2015.

[10] S. J. R. Coppens, Z. Zawodny, G. Dewinter, A. Neyrinck, A. L. Balocco, and S. Rex, "In search of the Holy Grail: poisons and extended release local anesthetics," Best Pract. Res. Clin. Anaesthesiol., vol. 33, pp. 3-21, 2019.

[11] M. J. Hughes, N. T. Ventham, S. McNally, E. Harrison, and S. Wigmore, "Analgesia after open abdominal surgery in the setting of enhanced recovery surgery: a systematic review and meta-analysis," JAMA Surg., vol. 149, pp. 1224-1230, 2014.

[12] J. Ripollés-Melchor ."Association between use of enhanced recovery after surgery protocol and postoperative complications in colorectal surgery: the postoperative outcomes within enhanced recovery after surgery protocol
(POWER) study," JAMA Surg., vol. 154, pp. 725-736, 2019.

[13] R. A. Moore, S. Derry, D. Aldington, and P. J. Wiffen, "Single dose oral analgesics for acute postoperative pain in adults-an overview of Cochrane reviews," Cochrane Database Syst. Rev., vol. 3, pp. 89-102, 2015.

[14] J. L. Apfelbaum, C. Chen, S. S. Mehta, and T. J. Gan, "Postoperative pain experience: results from a national survey suggest postoperative pain continues to be undermanaged," Anesth. Analg., vol. 97, pp. 534-540, 2003.

[15] S. Bell, T. Rennie, C. A. Marwick, and P. Davey, "Effects of peri-operative nonsteroidal anti-inflammatory drugs on post-operative kidney function for adults with normal kidney function," Cochrane Database Syst. Rev., vol. 5, pp. 110-121, 2018.

[16]C. R. Thangaswamy, V. Rewari, A. Trikha, and M. Dehran, "Dexamethasone before total laparoscopic hysterectomy: a randomized controlled dose-response study," J. Anesth., vol. 24, pp. 24-30, 2010.

[17] S. Shafi ."Association of opioid-related adverse drug events with clinical and cost outcomes among surgical patients in a large integrated health care delivery system," JAMA Surg., vol. 153, pp. 757-763, 2018

[18] J. Quinlan and F. Cox, "Acute pain management in patients with drug dependence syndrome," Pain reports, vol. 2, pp. 65-76, 2017.

[19] S. M. Nimmo and L. S. Harrington, "What is the role of epidural analgesia in abdominal surgery?," Contin. Educ. Anaesthesia, Crit. Care Pain, vol. 14, pp. 224-229, 2014.

[20] J. H. Salicath, E. C. Y. Yeoh, and M. H. Bennett, "Epidural analgesia versus patientcontrolled intravenous analgesia for pain following intra-abdominal surgery in adults," Cochrane Database Syst. Rev., vol. 4, pp. 78-95, 2018.

[21]D. E. Low ."Guidelines for perioperative care in esophagectomy: enhanced recovery after surgery (ERAS $\AA)$ society recommendations," World J. Surg., vol. 43, pp. 299-330, 2019.

[22] U. O. Gustafsson ."Guidelines for perioperative care in elective colorectal surgery: Enhanced Recovery After Surgery (ERAS®) Society recommendations: 2018," World J. Surg., vol. 43, pp. 659-695, 2019.

[23]P. I. Emelife . "Adjunct medications for peripheral and neuraxial anesthesia," Best Pract. Res. Clin. Anaesthesiol., vol. 32, pp. 83-99, 2018.

[24] G. Nelson ."Guidelines for perioperative care in gynecologic/oncology: Enhanced Recovery After Surgery (ERAS) Society recommendations-2019 update," Int. J. Gynecol. Cancer, vol. 29, pp. 80-91, 2019.

[25] J. L. Mosso Vázquez, D. Mosso Lara, J. L. 
Mosso Lara, I. Miller, M. D. Wiederhold, and B. K. Wiederhold, "Pain distraction during ambulatory surgery: virtual reality and mobile devices," Cyberpsychology, Behav. Soc. Netw., vol. 22, pp. 15-21, 2019. 